\title{
Nach Hirnblutung wieder antikoagulieren
}

\author{
Sollten Patienten, die unter einer Antikoagulation eine intrakranielle Blutung erlitten haben, die \\ Medikamente danach weiter nehmen? Eine Metastudie sieht in vielen Fällen eindeutige Vorteile.
}

\begin{abstract}
Acht Studien mit insgesamt 5.306 Patienten mit intrakraniellen Blutungen unter oraler Antikoagulation konnten ausgewertet werden. Vier Arbeiten schlossen nur Patienten mit parenchymatösen Hirnblutungen ein, die übrigen auch Patienten mit Subduralhämatomen und Subarachnoidalblutungen. Endpunkte waren ischämischer Schlaganfall und Myokardinfarkt sowie erneute intrakranielle Blutungen. Die häufigste Indikation für die Antikoagulation war Vorhofflimmern.

Wenn die Antikoagulation wieder aufgenommen wurde, geschah dies zwischen den Tagen 10 und 39 - in sieben der acht Studien mit einem Vitamin-KAntagonisten.
\end{abstract}

Thromboembolische Komplikationen wurden in sechs der Studien mit insgesamt 2.044 Patienten untersucht. Bei $38,4 \%$ von ihnen wurde die Antikoagulation erneut gestartet. Unter diesen lag die Rate thromboembolischer Ereignisse bei $6,7 \%$, in der Gruppe ohne Antikoagulation dagegen bei $17,6 \%$.

Das Risiko einer erneuten intrakraniellen Blutung wurde in allen acht Studien ermittelt. 35,8\% der Probanden wurden erneut antikoaguliert. Ihre Blutungsrate lag bei $8,7 \%$, in der Gruppe ohne Antikoagulation betrug sie 7,8\%.

\footnotetext{
- Murthy SB, Gupta A, Merkler AE et al. Restarting anticoagulant therapy after intracranial hemorrhage: a systematic review and meta-analysis. Stroke 2017, online 17. April; https:// doi.org/10.1161/STROKEAHA.116.016327
}

\begin{abstract}
KOMMENTAR
Diese Analyse ist hilfreich, da sie zeigt, dass man bei bis zu $40 \%$ aller betroffenen Patienten die Antikoagulation zu einem späteren Zeitpunkt wieder starten kann. Wenn dies mit Blick auf das Blutungsrisiko möglich ist, haben diese $\mathrm{Pa}$ tienten einen eindeutigen Nutzen bezüglich der Verhinderung weiterer thromboembolischer Ereignisse. Die Kohortenstudien sind natürlich nicht randomisiert. Bei $60 \%$ der Patienten haben die Ärzte offenbar das Risiko einer erneuten Hirnblutung für zu hoch eingeschätzt. Zu der Frage lohnt sich eine sorgfältig geplante und durchgeführte randomisierte Studie.
\end{abstract}

Prof. Dr. med. H.-C. Diener

\section{Das mit dem Knallfrosch ist ins Auge gegangen}

Beim Zünden eines Feuerwerkskörpers hatte sich ein 44-jähriger Mann schwere Augenverletzungen zugezogen. Bei der Vorstellung in der Fachklinik klagte er über Schmerzen in den Augen und einen vollständigen Visusverlust rechts. Am linken Auge ergab die Untersuchung eine Visusminderung auf 20/80.

Auf dem rechten Auge bestand eine perforierende Verletzung. Die Spaltlampen-Untersuchung des linken Auges zeigte multiple Fremdkörper, die zum Teil oberflächlich, zum Teil in der Tiefe der Hornhaut lagen (Abb. A). Auf dem CT beider Orbitae stellten sich weitere intraokulär gelegene Fremdkörper dar (Abb. B). Zu einem solchen Eindringen kommt es durch Akzelerationsverletzungen des Auges, z. B. infolge von Explosionen, Schüssen aus Feuerwaffen oder Unfällen am Arbeitsplatz. Daher muss bei Arbeiten, bei denen harte Materialteile durch die Luft fliegen können, immer eine Schutzbrille getragen werden.

Leider ging die Sache für den Mann nur zum Teil gut aus. Die Fremdkörper wurden chirurgisch aus der Hornhaut entfernt. Zur Infektionsprophylaxe gab man Antibiotika und künstliche Augen-
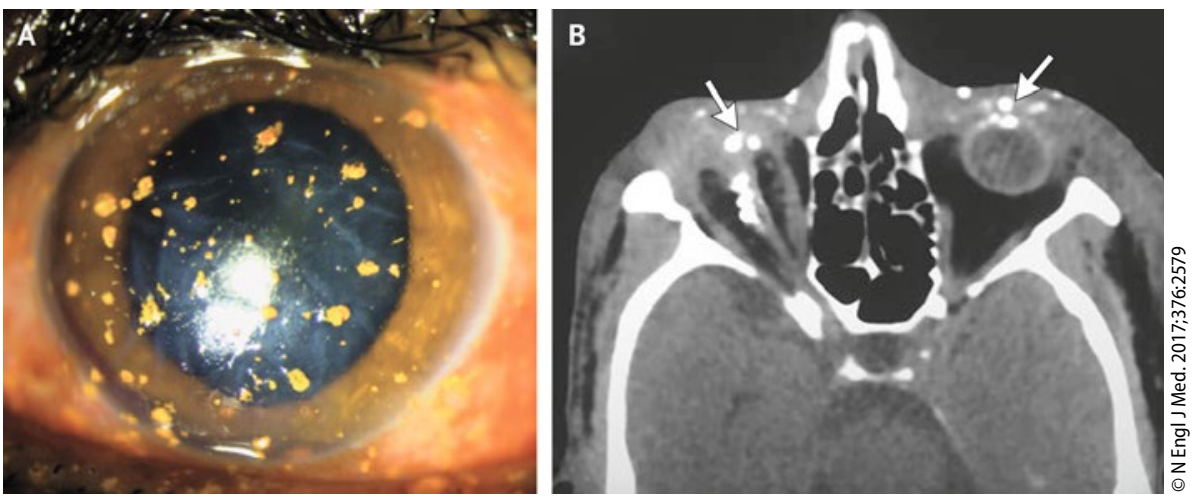

A: Multiple Fremdkörper in der Hornhaut des linken Auges. B: Intraokuläre Fremdkörper im axialen CT (Pfeile). tropfen. Im Laufe der nächsten drei Monate verbesserte sich der Visus am linken Auge auf 20/40. Schlimmer war es um das rechte Auge bestellt. Trotz einer Operation blieb es dauerhaft funktionslos und fing an zu schrumpfen - ein Zustand, der etwas blumig als „Phthisis bulbi“ bezeichnet wird.

Prof. Dr. med. H. S. FüeßI

- Gupta PC, Ram J. Eye injury from firecracker. NEngl J Med. 2017;376:2579 